\title{
Influence of Low-Level, High-Entropy Air in the Eye on Tropical Cyclone Intensity: A Trajectory Analysis
}

\author{
Xingyang ZHOU, Liguang WU \\ Department of Atmospheric and Oceanic Sciences and Institute of Atmospheric Sciences, Fudan University, China \\ State Key Laboratory of Severe Weather, Chinese Academy of Meteorological Sciences, China
}

Qingyuan LIU

Jiangsu Institute of Meteorological Sciences, China

and

Yan ZHENG

Key Laboratory of Meteorological Disaster of Ministry of Education, Nanjing University of Information Science and Technology, China

(Manuscript received 14 October 2019, in final form 22 July 2020)

\begin{abstract}
As suggested by previous studies, the entrainment of the low-level high-entropy eye air can provide additional energy for tropical cyclone (TC) intensification, but the previous trajectory analysis only indicated that considerable air parcels below the eye inversion could be entrained into the eyewall. In the present study, the $1 \mathrm{~min}$ output data from a semi-idealized experiment are utilized to quantitatively evaluate the relative importance of the entrainment of the high-entropy eye air by enhancing the eyewall convection.

It is confirmed that a significant amount of high-entropy eye air below the eye inversion can be entrained into the eyewall. The entrainment occurs favorably on the quadrants of enhanced eyewall convection and is enhanced in the presence of small-scale disturbances in the inner edge of the eyewall. However, the eyewall air parcels below $3 \mathrm{~km}$ experience a fast cycling. There are $84.4 \%$ and $7.7 \%$ eyewall air from the low-level inflow and the middle-level dry environment, respectively. The low-level high-entropy eye air only accounts for $1.7 \%$ of the eyewall air, whereas $6.2 \%$ eyewall air remains in the eyewall below $3 \mathrm{~km}$ during the $90 \mathrm{~min}$ period. The eye air from the low-level high-entropy reservoir accounts for $5.8 \%$ of the equivalent potential temperature change below $3 \mathrm{~km}$ and $4.5 \%$ of the total mass transport at $3 \mathrm{~km}$ in the TC eyewall. The present study suggests that the low-level high-entropy air from the eye has little direct influence on TC intensity through enhancing the eyewall convection by providing relatively small mass and thermodynamic contributions.
\end{abstract}

Keywords high-resolution numerical simulation; tropical cyclone intensity; trajectory analysis

Corresponding author: Liguang Wu, Department of Atmospheric and Oceanic Sciences and Institute of Atmospheric Sciences, Fudan University, Room 5007, Environmental Science Building, No. 2005 Songhu Road, Shanghai 200438, China

E-mail: liguangwu@fudan.edu.cn

J-stage Advance Published Date: 28 August 2020

(C) The Author(s) 2020. This is an open access article published by the Meteorological Society of Japan under a Creative Commons Attribution 4.0 International (CC BY 4.0) license (https://creativecommons.org/licenses/by/4.0). 
Citation Zhou, X., L. Wu, Q. Liu, and Y. Zheng, 2020: Influence of low-level, high-entropy air in the eye on tropical cyclone intensity: A trajectory analysis. J. Meteor. Soc. Japan, 98, 1231-1243, doi:10.2151/jmsj.2020-063.

\section{Introduction}

An inversion exists in mature tropical cyclone (TC) eyes, which separates the low-level high-entropy air from the warm, dry air aloft (Willoughby 1998; Montgomery et al. 2006). It is suggested that the high-entropy eye air is an additional source of energy for TC intensification through mixing with the eyewall air (Persing and Montgomery 2003; Cram et al. 2007; Montgomery et al. 2006; Bell and Montgomery 2008; Barnes and Fuentes 2010; Miyamoto and Takemi 2013), which may make the TC intensity stronger than the maximum potential intensity (MPI) predicted by Emanuel (1986, 1995). Although the MPI theory is independent of the eye dynamics, Emanuel (1997) suggested that TC intensification can be indirectly accelerated by turbulent stresses that occur in the eye and eyewall. Bryan and Rotunno (2009) found that the influence of the low-level high-entropy eye air on steady-state maximum TC intensity was negligible in their numerical simulation of an axisymmetric TC model. Wang and Heng (2016) indicated that the effect of the high-entropy eye air on TC intensity was not through the modifications to the overall strength of eyewall convection. Presently, whether the highentropy eye air can substantially increase TC intensity through mixing with eyewall air and enhancing eyewall convection remains a scientific issue.

The peak intensity of simulated TCs in very high-resolution models often exceeds the MPI (Persing and Montgomery 2003; Cram et al. 2007; Yang et al. 2007; Bryan and Rotunno 2009; Xu and Wang 2010a, b). Persing and Montgomery (2003) was the first to propose the influence of the high-entropy air below the inversion of the eye on TC intensity. They found that the TC intensity simulated in an axisymmetric, cloud-resolving model with high spatial and temporal resolutions exceeded the upper limit predicted by the MPI theory (Emanuel 1986, 1995). Since the so-called superintensity occurred only in the presence of the enhanced low-level entropy in the eye of the simulated TC, Persing and Montgomery (2003) hypothesized that the superintensity was due to the impact of the entrainment of the low-level high-entropy air from the eye to the eyewall. The MPI theory assumes that no entropy is fluxed from the eye to the eyewall. Cram et al. (2007) examined the entrainment via an analysis of air parcel trajectories. Their analysis was based on the cloud-resolved simulation of Hurricane Bonnie (1998) conducted in Braun et al. (2006). Cram et al. (2007) confirmed the entrainment of the high-entropy air below the inversion of the TC eye to the eyewall, but they did not evaluate the relative importance of the high-entropy air that is entrained into the eyewall.

The hypothesis was further examined in Huirricane Isabel (2003), in which the strongest horizontal wind of $107 \mathrm{~m} \mathrm{~s}^{-1}$ was observed at $1.4 \mathrm{~km}$ altitude by a National Center for Atmospheric Research GPS dropsonde (Aberson et al. 2006). Using the observed data in Isabel, Montgomery et al. (2006) found that the observed azimuthal mean tangential wind speed in the boundary layer $\left(76 \mathrm{~m} \mathrm{~s}^{-1}\right.$, with a $6 \mathrm{~m} \mathrm{~s}^{-1}$ standard deviation) was much stronger than the theoretically predicted $56.6 \mathrm{~m} \mathrm{~s}^{-1}$ MPI. They argued that the entrainment of the enhanced low-level entropy air from the eye to the eyewall proposed by Persing and Montgomery (2003) was the most likely candidate to explain the superintensity of Hurricane Isabel (2003).

The above studies did not address whether the low-level high-entropy air in the eye can substantially increase the maximum intensity of TCs by enhancing the eyewall convection although the entrainment of the low-level high-entropy air from the eye to the eyewall is confirmed. Using a time-dependent axisymmetric numerical model, Bryan and Rotunno (2009) indicated that the enhanced low-level entropy in the TC eye is created mainly by surface entropy fluxes. This is consistent with the trajectory analysis in Cram et al. (2007). Cram et al. (2007) found that a portion of the low-level inflow bypasses the eyewall to enter the eye and lingers for about $1 \mathrm{~h}$ to acquire enhanced entropy characteristics through interaction with the ocean beneath the eye. For this reason, the effect of the low-level high-entropy air in the eye can be examined by setting the surface fluxes to 0 in the numerical experiment. Bryan and Rotunno (2009) turned off the enthalpy flux from the sea surface in the eye while the angular momentum mixing effect remained and found that the axisymmetric tangential wind speed is only slightly weakened by about $4 \%$ in their experiment without the surface fluxes. Their further analysis indicated that less than $3 \%$ of the total surface entropy input to the TC comes from the eye. This was confirmed by Wang and Xu (2010) through sensitivity 
experiments in the three-dimensional cloud-resolving model simulations. Bryan and Rotunno (2009) concluded that the transport of the high-entropy air below the inversion from the TC eye into the eyewall has little impact on the maximum axisymmetric intensity of TCs. Wang and Heng (2016) found that the nearsurface high-entropy air in the eye region can initiate convection near the inner edge of the eyewall and then facilitate eyewall contraction, leading to higher innercore inertial stability and then increasing TC intensification rate.

We notice that the conclusion of Bryan and Rotunno (2009) was based on the numerical simulation of an axisymmetric numerical model. The trajectory analysis in Cram et al. (2007) indicated that there was an overall tendency for the trajectories to be stirred out into the eyewall down-shear and left of vertical wind shear, suggesting the influence of the asymmetric structure of the TC on the transport. Additionally, mesoscale and microscale vortices are usually observed in the inner region of intense TCs, which are closely related to the vortex Rossby wave. The mesoscale vortices can cause the high potential vorticity of the eyewall to stir into the eye (Black and Marks 1991; Kossin et al. 2002; Knaff et al. 2003), which may affect the entrainment of the enhanced low-level entropy air from the eye to the eyewall. Aberson et al. (2006) suggested that the entrainment of the low-level high-entropy air from the eye to the eyewall in Hurricane Isabel (2006) can be enhanced in the presence of the small-scale or tornado-scale vortex, which usually occurs at low levels in the inner edge of the intense eyewall convection (Marks et al. 2008; Aberson et al. 2017; Ito et al. 2017; Wurman and Kosiba 2018). Using the large eddy simulation (LES) in the Advanced Weather Research and Forecast (WRF) model, Wu et al. $(2018,2019)$ recently conducted a numerical experiment including seven nesting domains with the finest horizontal grid size of $37 \mathrm{~m}$ and confirmed the existence of tornado-scale vortices in the turbulent boundary layer of the TC eyewall and indicated that tornado-scale vortices are prevalent in the inner edge of the intense eyewall convection. The axisymmetric models cannot generate the mesoscale to microscale vortices. It is suggested that the influence of the low-level high-entropy air in the eye on TC intensity should be examined in a more realistic TC than the axisymmetric numerical model.

Clearly, the relative importance of the low-level, high-entropy to eyewall convection has not been fully understood in the previous trajectory analysis (Cram et al. 2007). Additionally, the numerical experiments cannot separate the relative contribution from other influences such as the induced eyewall contraction and angular momentum mixing (Bryan and Rotunno 2009; Wang and Heng 2016). In the present study, the output data from the high-resolution numerical simulation of a semi-idealized TC that evolves in a realistic largescale environment are used to examine the relative contribution of the low-level high-entropy eye air as an additional source of energy through mixing with the eyewall air, with a focus on the influence of smallscale structures on the entrainment from the eye to the eyewall. The simulated TC is described in Section 2, whereas the analysis of the entrainment of low-level high-entropy air in the eye to the eyewall and the possible influence of the asymmetric structure are discussed in Section 3. In Section 4, the origins of the eyewall air and the influence of low-level high-entropy air in the eye are examined, followed by a summary in Section 5.

\section{The simulated TC}

The TC data used in the present study are based on a numerical experiment, which resembles that in $\mathrm{Wu}$ et al. $(2018,2019)$ except the coarser grid size of the innermost domain. The experiment was conducted with the version 3.2 .1 of the WRF model, but the horizontal grid size of the innermost domain is $1 / 3 \mathrm{~km}$ in the present study. The outermost domain centered at $30.0^{\circ} \mathrm{N}, 132.5^{\circ} \mathrm{E}$ covered an area of $6210 \times 6210$ $\mathrm{km}^{2}$ and contained $230 \times 210$ grid points with $27 \mathrm{~km}$ grid spacing. The four nested, two-way interactive domains contained $230 \times 210,432 \times 399,333 \times 333$, and $721 \times 721$ grid points, respectively. The corresponding horizontal grid sizes were $9,3,1$, and $1 / 3$ $\mathrm{km}(\sim 333 \mathrm{~m})$, respectively. The 3,1 , and $1 / 3 \mathrm{~km}$ grid domains followed the movement of simulated storm. The model top was $50 \mathrm{hPa}$ with 75 vertical levels. The vertical grid size ranged from $70-100 \mathrm{~m}$ below $1 \mathrm{~km}$ to $250-400 \mathrm{~m}$ above $1 \mathrm{~km}$.

Following $\mathrm{Wu}$ and Chen (2016), a symmetric vortex was spun up for $18 \mathrm{~h}$ on an f-plane in a resting environment, and then, the vortex was placed in the largescale background of Typhoon Matsa (2005) from 0000 UTC 5 August to 1200 UTC 6 August. The largescale environment was from the National Centers for Environmental Prediction Final Operational Global Analysis data with the grid size of $1.0^{\circ} \times 1.0^{\circ}$. The low-frequency environment was obtained with a 20 day low-pass Lanczos filter (Duchon 1979). The Kain-Fritsch cumulus parameterization scheme and the WRF single-moment three-class microphysics scheme was used in the outermost domain (Kain 

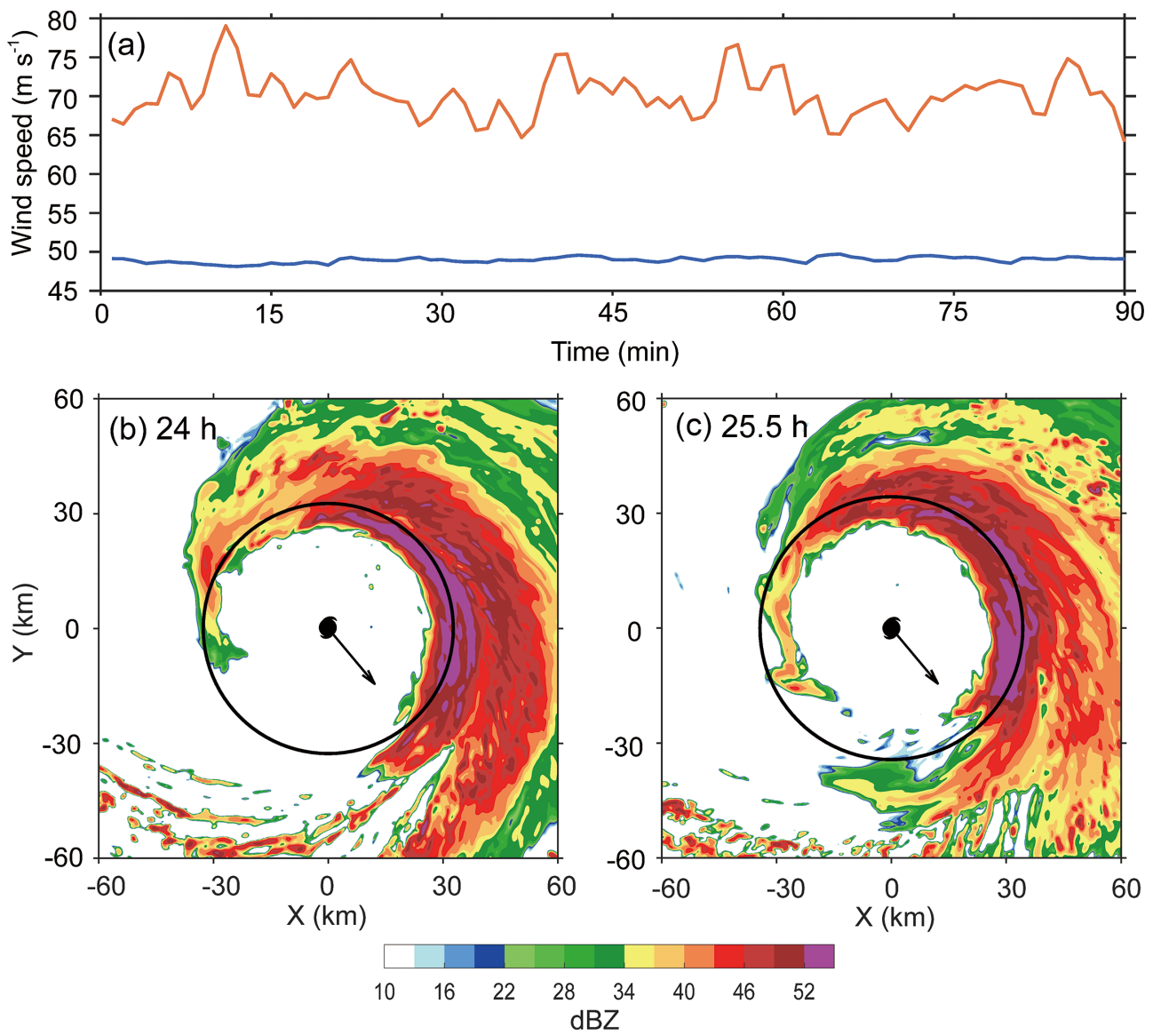

Fig. 1. (a) Intensity of the simulated tropical cyclone during $24-25.5 \mathrm{~h}$ in terms of instantaneous (orange) and azimuthal maximum (blue) wind speeds at $10 \mathrm{~m}$. (b, c) The simulated $3 \mathrm{~km}$ radar reflectivity (dBZ) at $24 \mathrm{~h}$ (left) and $25.5 \mathrm{~h}$ (right). The black circles in (b) and (c) indicate the $10 \mathrm{~m} \mathrm{RMW}$ at $24 \mathrm{~h}(32.7 \mathrm{~km})$ and $25.5 \mathrm{~h}(34.3 \mathrm{~km})$, whereas the arrow indicates the vector of vertical wind shear $\left(7.0 \mathrm{~m} \mathrm{~s}^{-1}\right.$ at $24 \mathrm{~h}$ and $6.9 \mathrm{~m} \mathrm{~s}^{-1}$ at $\left.25.5 \mathrm{~h}\right)$.

and Fritsch 1993), whereas the WRF single-moment six-class microphysics scheme was used in the four nested domains (Hong and Lim 2006). The option of the LES simulation was used in the innermost domain (Mirocha et al. 2010), and the Yonsei University PBL parameterization scheme (Noh et al. 2003) was used in the other domains.

The simulation was run for $72 \mathrm{~h}$ over the open ocean with the uniform sea surface temperature of $29^{\circ} \mathrm{C}$. Although the model output is regularly at $1 \mathrm{~h}$ intervals, for the purpose of trajectory calculation, we set the output at $1 \mathrm{~min}$ intervals during a $90 \mathrm{~min}$ period $(24-25.5 \mathrm{~h})$. Figure 1a shows the intensity of the simulated TC in terms of instantaneous and azimuthal maximum wind speeds at $10 \mathrm{~m}$ during the $90 \mathrm{~min}$ period. Although the instantaneous maximum wind speed is between $64.8 \mathrm{~m} \mathrm{~s}^{-1}$ and $78.6 \mathrm{~m} \mathrm{~s}^{-1}$, the azimuthal wind speed ranges from $46.5 \mathrm{~m} \mathrm{~s}^{-1}$ to $48.8 \mathrm{~m} \mathrm{~s}^{-1}$. We can see that the TC intensity generally fluctuates around the average over the period. Note that the azimuthal maximum wind speed reaches the peak around $12 \mathrm{~h}$ and remains relatively steady during $18-42 \mathrm{~h}$.

Figures $1 \mathrm{~b}$ and $1 \mathrm{c}$ show the simulated radar reflectivity at the $3 \mathrm{~km}$ altitude in the TC inner-core region and the vector of vertical wind shear at $24 \mathrm{~h}$ and $25.5 \mathrm{~h}$. The vertical wind shear is calculated as the wind differences between $200 \mathrm{hPa}$ and $850 \mathrm{hPa}$ over a radius of $500 \mathrm{~km}$. The vertical wind shear is northwesterly with a magnitude of about $7 \mathrm{~m} \mathrm{~s}^{-1}$. In agreement with previous studies (e.g., Frank and Ritchie 1999), the enhanced eyewall convection occurs on the downshear left side, whereas the eyewall of the simulated TC is not closed. During the 90 min period, the sim- 

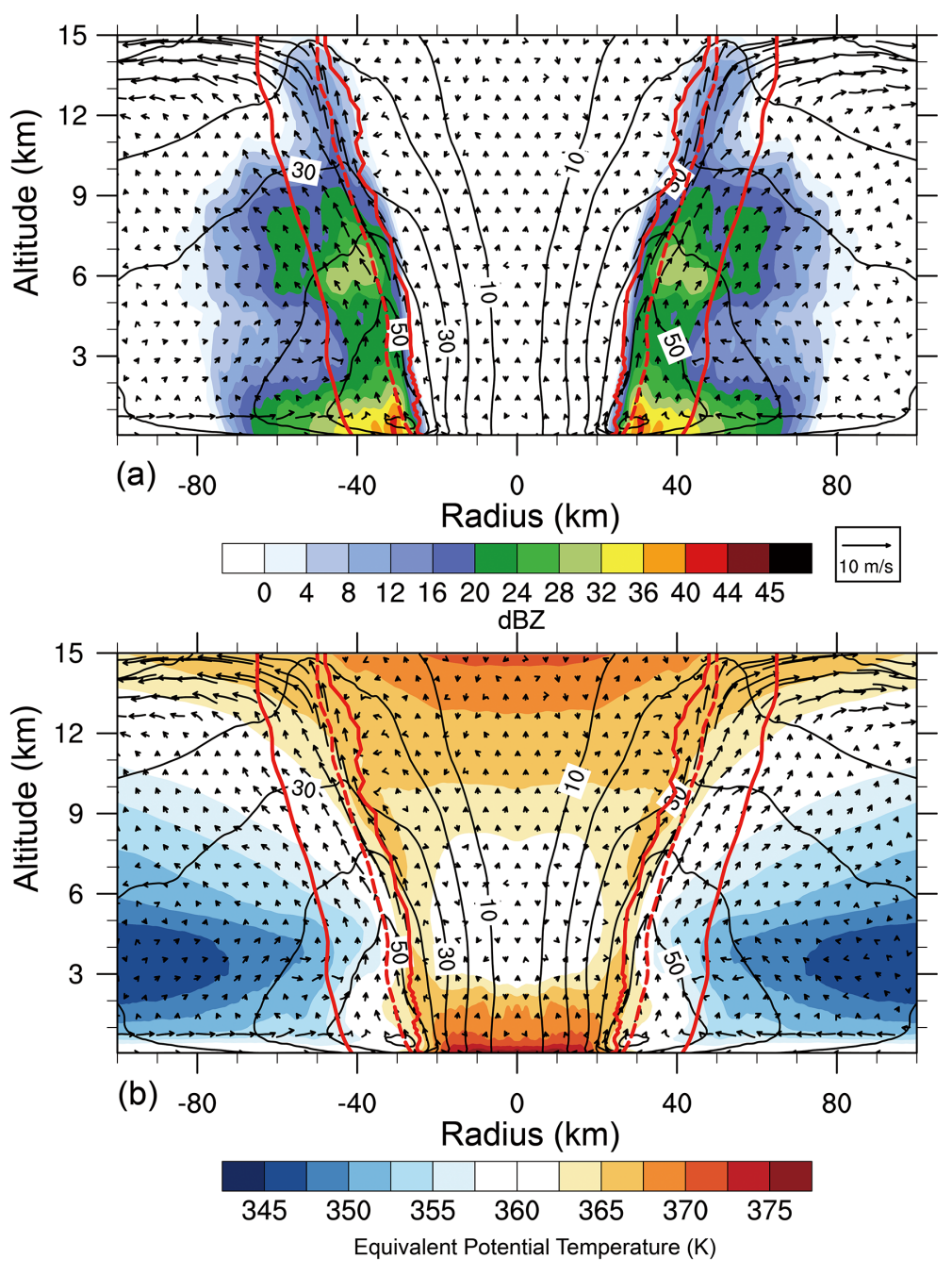

Fig. 2. (a) Vertical cross-sections of azimuthally averaged vertical and radial winds ( $\mathrm{m} \mathrm{s}^{-1}$, vector), radar reflectivity (dBZ, shading), and temperature difference from the average over the radius of $500 \mathrm{~km}$ from the $\mathrm{TC}$ center $\left({ }^{\circ} \mathrm{C}\right.$, contour) at $24 \mathrm{~h}$, the $\mathrm{x}$-axis indicates the distance to the center of the TC in kilometers; (b) the same as (a) but for vertical and radial winds ( $\mathrm{m} \mathrm{s}^{-1}$, vector), equivalent potential temperature (shading, $\mathrm{k}$ ), and azimuthal mean tangential wind speed $\left(\mathrm{m} \mathrm{s}^{-1}\right.$, contour). The solid lines denote the inner and outer boundaries of the eyewall with the dashed lines indicating the RMW.

ulated TC is at an observational steady-state because of the shear-induced asymmetry during this $90 \mathrm{~min}$ period. The influence of the asymmetric structure of the simulated TC on the entrainment of the highentropy air into the eyewall will be discussed in the next section.

Figure $2 \mathrm{a}$ shows the vertical cross-section of vertical and radial winds, the simulated radar reflectivity, and the temperature anomalies relative to the environment at $24 \mathrm{~h}$. The warm core structure and the high-entropy reservoir at the low levels are clearly shown in this figure. The eyewall is indicated by the enhanced reflectivity and strong eyewall updrafts. At this time, the near-surface radius of maximum wind (RMW) is $32.7 \mathrm{~km}$. The low-level inflow below $\sim 1.5$ $\mathrm{km}$ converges to the eyewall updraft, which extends radially outward to about $13 \mathrm{~km}$ and turns into the outflow. Following Stern and Zhang (2013), the warm core is defined based on the mean environmental temperature averaged over the 500-1000 km annulus. The altitude of the warm core with a maximum of about $11.2^{\circ} \mathrm{C}$ at $7.5 \mathrm{~km}$ is consistent with the numer- 
ical simulation of Stern and Nolan (2012) and Stern and Zhang (2013).

Figure $2 \mathrm{~b}$ shows the vertical cross-section of the azimuthal mean tangential wind speed and equivalent potential temperature $\left(\theta_{e}\right)$ at $24 \mathrm{~h}$. The strongest tangential wind occurs at about $500 \mathrm{~m}$. In agreement with the previous observations (Jordan 1952; Willoughby 1998), the low-level $\theta_{e}$ in the eye is enhanced with a maximum of $377 \mathrm{~K}$, forming a high-entropy reservoir in the low-level TC eye. The eyewall region is also plotted in Fig. 2. The inner boundary of the eyewall is defined by the azimuthal mean vertical motion of 0.5 $\mathrm{m} \mathrm{s}^{-1}$, whereas the outer boundary is $10 \mathrm{~km}$ radially outward from the RMW. The threshold of the azimuthal mean vertical motion is larger than that used in Cram et al. (2007) because of higher horizontal resolution in our simulation. The defined region generally covers the strong vertical motion with the most active convection in the eyewall. As shown in Fig. 2b, the $\theta_{e}$ in the eye can exceed that in the eyewall by more than $10 \mathrm{~K}$.

The high-entropy reservoir in the low-level TC eye is accompanied by a low-level inversion, which is clear in the skew-T Log-P diagram of the sounding at the TC center (Fig. 3a). The inversion with a bottom between $850 \mathrm{hPa}$ and $700 \mathrm{hPa}$ separates the dry, warm air aloft from moist air near the surface in the eye (Jordan 1952; Willoughby 1998). Above the bottom of the inversion, $\theta_{e}$ decreases first and then increases with height. The minimum value is about $359 \mathrm{~K}$ at an altitude of $5 \mathrm{~km}$ in the eye.

\section{Trajectories of the low-level high-entropy air parcels in the eye}

The method for the trajectory calculation in the present study was adopted from the WRF postprocessing RIP4 software (which stands for Read/ Interpolate/Plot). Since the innermost domain moves with the TC center at an interval of $15 \mathrm{~min}$, the $90 \mathrm{~min}$ output data at $1 \mathrm{~min}$ intervals are first transformed into a fixed reference framework. Note that the resulting forward trajectories are plotted in the reference framework moving with the TC center. The TC center in the present study was determined with the method for the minimum pressure variance center (Braun 2002; Braun et al. 2006). At a specific initial moment and height ( $z$ coordinate), the trajectory starting locations are evenly seeded with a $1 \mathrm{~km}$ spacing in both zonal and meridional directions. The high-entropy reservoir is defined within the inner boundary of the eyewall below $3 \mathrm{~km}$ in the eye. More than $99 \%$ air parcels that are initially in the eye have a higher $\theta_{e}$ than that
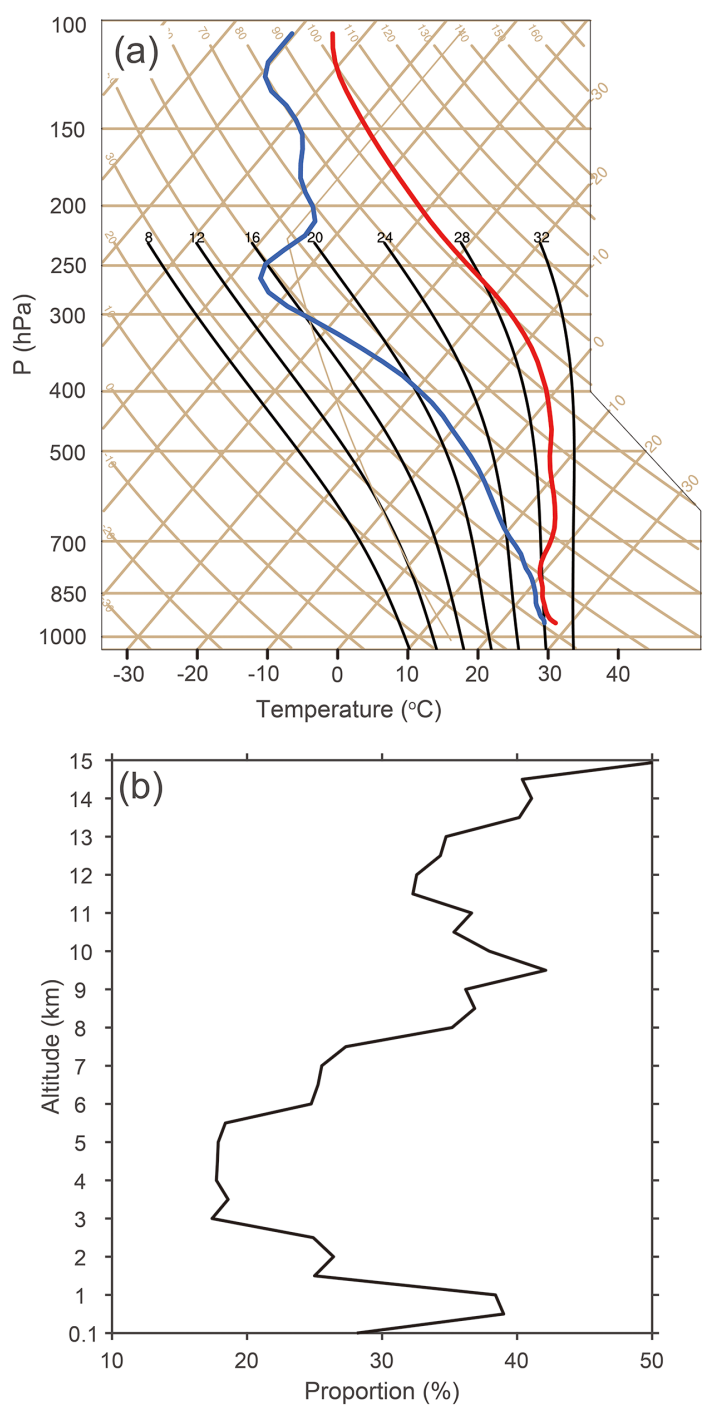

Fig. 3. (a) Skew-T Log-P diagram of the eye sounding at $24 \mathrm{~h}$. The red and blue lines are the vertical profiles of temperature profile $\left({ }^{\circ} \mathrm{C}\right)$ and dewpoint $\left({ }^{\circ} \mathrm{C}\right)$, respectively. (b) The percentage of eye air parcels that enter the eyewall as a function of altitude within $90 \mathrm{~min}$.

averaged over the eyewall region.

Table 1 shows the number of the air parcels initially in the TC eye as a function of height between $0.1 \mathrm{~km}$ and $15 \mathrm{~km}$. Since the eye area varies with height, the initial number of the air parcels generally increases with height. The percentage of the air parcels that enter the eyewall region is calculated from $0.1 \mathrm{~km}$ to $15 \mathrm{~km}$ during the $90 \mathrm{~min}$ period (Fig. $3 \mathrm{~b}$ ). At the lower levels, as shown in Fig. 3b, the percentage reaches 
Table 1. List of the number of initial air parcels in the eye and eyewall at altitudes $(\mathrm{km})$ between 0.1 $\mathrm{km}$ and $15 \mathrm{~km}$.

\begin{tabular}{|c|c|c|c|c|c|}
\hline Altitude & Eye parcels & Eyewall parcels & Altitude & Eye Parcels & Eyewall Parcels \\
\hline 0.1 & 1909 & 2291 & 8 & 3732 & 3958 \\
\hline 0.5 & 1864 & 2692 & 8.5 & 3934 & 4133 \\
\hline 1 & 2068 & 2795 & 9 & 3924 & 4461 \\
\hline 1.5 & 1856 & 3220 & 9.5 & 4706 & 3960 \\
\hline 2 & 2117 & 3153 & 10 & 4659 & 4338 \\
\hline 2.5 & 2289 & 3253 & 10.5 & 4988 & 4460 \\
\hline 3 & 2177 & 3543 & 11 & 5274 & 4560 \\
\hline 3.5 & 2253 & 3454 & 11.5 & 5347 & 4587 \\
\hline 4 & 2256 & 3375 & 12 & 5394 & 4600 \\
\hline 4.5 & 2291 & 3394 & 12.5 & 5664 & 4639 \\
\hline 5 & 2351 & 3556 & 13 & 6089 & 4551 \\
\hline 5.5 & 2441 & 3735 & 13.5 & 6321 & 4473 \\
\hline 6 & 2788 & 3664 & 14 & 6363 & 4415 \\
\hline 6.5 & 2942 & 3750 & 14.5 & 6342 & 4308 \\
\hline 7 & 3082 & 3860 & 15 & 6350 & 4054 \\
\hline 7.5 & 3229 & 4063 & & & \\
\hline
\end{tabular}

a maximum of $39.1 \%$ at $0.5 \mathrm{~km}$ and decreases to a minimum of about $18 \%$ at $3 \mathrm{~km}$. At the middle and upper levels, the proportion generally increases with height with a maximum at $9.5 \mathrm{~km}(43.5 \%)$. Our analysis suggests that the increasing entrainment of the eye air into the eyewall at the middle and upper levels is associated with the enhanced eyewall convection. In the present study, we focus only on the entrainment of low-level high-entropy air in the TC eye. Despite the relatively short period used in the present study, our results are generally comparable with those in Cram et al. (2007). They found that the maximum percentage of $56.8 \%$ occurred at $453 \mathrm{~m}$ within the 5 $\mathrm{h}$ period. Consistent with Cram et al. (2007), here, we also confirm that a considerable proportion of the air parcels in the low-level high-entropy reservoir enter the eyewall region to mix with the eyewall air.

We find that the entrainment of the eye air parcels in the low-level high-entropy reservoir to the eyewall is confined to the layer below $3 \mathrm{~km}$ during the $90 \mathrm{~min}$ period. They are initially located less than $6 \mathrm{~km}$ away from the inner edge of the defined eyewall, mainly because of the lack of strong outflow below the lowlevel inversion. Figure 4 shows a subset of 15 air parcel trajectories (randomly selected to represent three typical groups) that are initially at $0.5 \mathrm{~km}$, representing the typical forward trajectories of the entrainment of the high-entropy eye air to the eyewall. Their initial $\theta_{e}$ ranges from $368 \mathrm{~K}$ to $372 \mathrm{~K}$ and they are entrained into the eyewall mainly between the altitudes of $0.5 \mathrm{~km}$ and $1.5 \mathrm{~km}$. After they enter the eyewall, their $\theta_{e}$ is close to that in the eyewall within $10 \mathrm{~min}$. The parcels can encounter the maximum updraft of $13.8 \mathrm{~m} \mathrm{~s}^{-1}$ and downdraft of $-9.1 \mathrm{~m} \mathrm{~s}^{-1}$. As shown in Fig. 4, the air parcel trajectories in the 90 min period can be roughly classified into three categories: ascending with the eyewall updraft $(56.5 \%$; T1) and even reaching the altitude of $13 \mathrm{~km}(3.1 \%)$, being detrained into the middle-level eye $(35.8 \%, \mathrm{~T} 2)$ and outside environment $(7.7 \%, \mathrm{~T} 3)$.

Cram et al. (2007) also found that the air parcels in the low-level high-entropy reservoir prefer to make their way out to the eyewall region with strong eyewall convection. Such a tendency is also found in the present study. Figure 5 shows the simulated radar reflectivity and where the eye air parcels initially at $0.5 \mathrm{~km}$ enter the eyewall. We can see that most of the air parcels are entrained into the eyewall on the side with enhanced eyewall convection. Figure 6 (blue bar) further shows the histograms of the azimuths of the points shown in Fig. 5. The peak is to the northeast of the TC center, corresponding to the most active convection in the eyewall.

Besides the asymmetric structure discussed above, recent studies confirmed the presence of the smallscale or tornado-scale vortex usually at low levels in the inner edge of the intense eyewall convection (Marks et al. 2008; Aberson et al. 2017; Ito et al. 2017; Wurman and Kosiba 2018; Wu et al. 2018, 2019). Aberson et al. (2006) suggested that the small-scale vortex may enhance the entrainment of the low-level high-entropy air from the eye to the eyewall in Isabel 

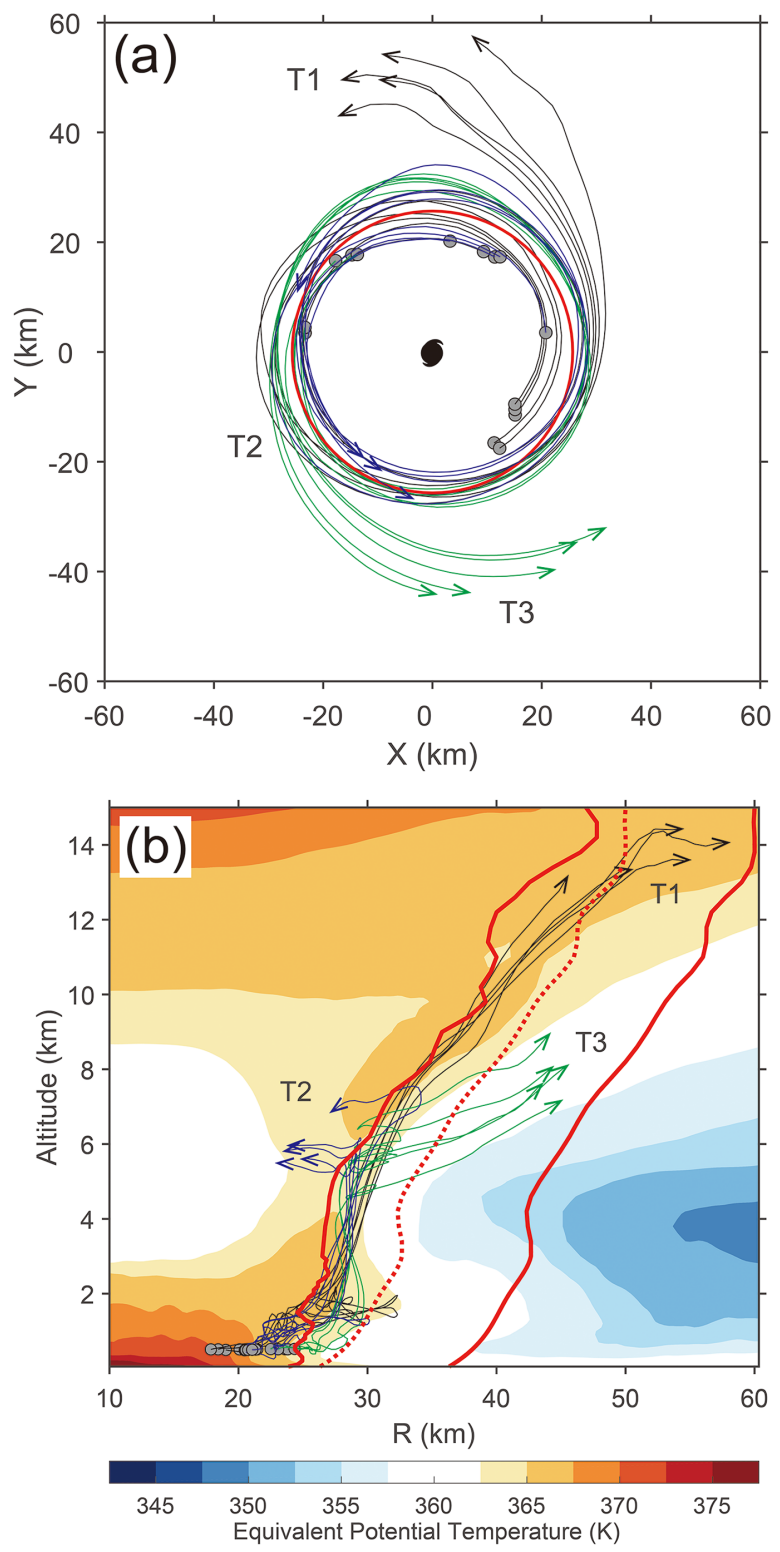

Fig. 4. 90 min trajectories for a subset of 15 eye air parcels initially at $0.5 \mathrm{~km}$ : (a) horizontal view and (b) vertical view. The circle in (a) and dashed line in (b) indicate the RMW, and the solid lines in (b) denote the inner and outer boundaries of the eyewall. T1 (black), T2 (blue), and T3 (green) represent three types of the trajectories: ascending with the eyewall updraft (T1), being detrained into the middle-level eye (T2) and outside environment (T3).

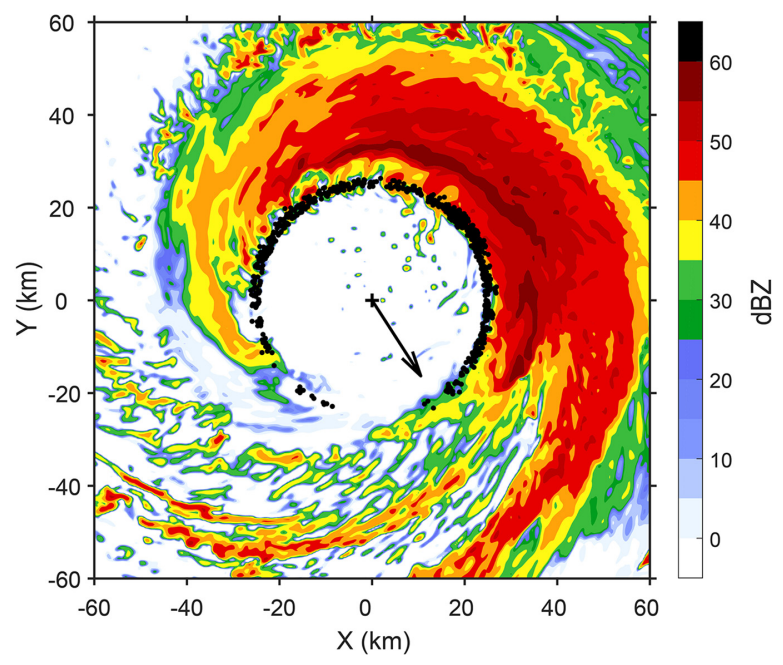

Fig. 5. Entrance locations of eye air parcels (black dots) initially at $0.5 \mathrm{~km}$ and enter the eyewall and the simulated $0.5 \mathrm{~km}$ radar reflectivity $(\mathrm{dBZ}$, shading) at $24 \mathrm{~h}$ with the vector of vertical wind shear (arrow).

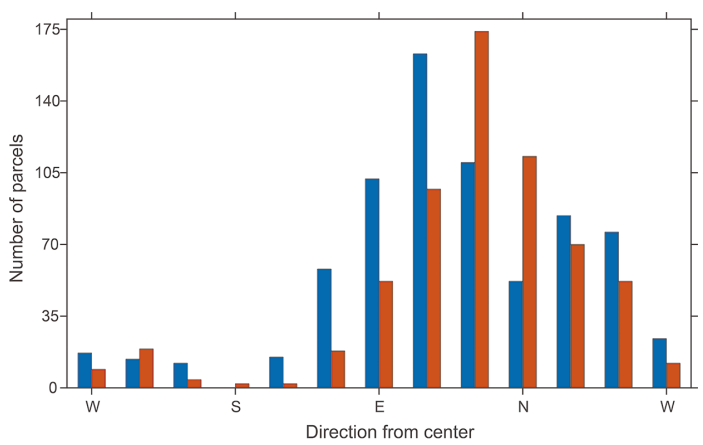

Fig. 6. Histogram of the azimuths of the eye air parcels that are initially at $0.5 \mathrm{~km}$ and then enter the eyewall. The blue and red bars indicate the parcel numbers for the original wind field (shown in Fig. 5) and the wind field with no perturbation components.

(2006). Figure $7 \mathrm{a}$ shows the $0.5 \mathrm{~km}$ wind vector and speed on the northern side of the TC at $24 \mathrm{~h} 45 \mathrm{~min}$. As shown in Fig. 1b, the eyewall convection is enhanced on the side with the strongest convection in the northeast quadrant. The maximum wind speed in Fig. $7 \mathrm{a}$ is $89.9 \mathrm{~m} \mathrm{~s}^{-1}$, and the maximum relative vertical vorticity is $0.07 \mathrm{~s}^{-1}$. The intense winds occur mainly inside the RMW. The streaks of alternating high and low wind speeds suggest the presence of small-scale 

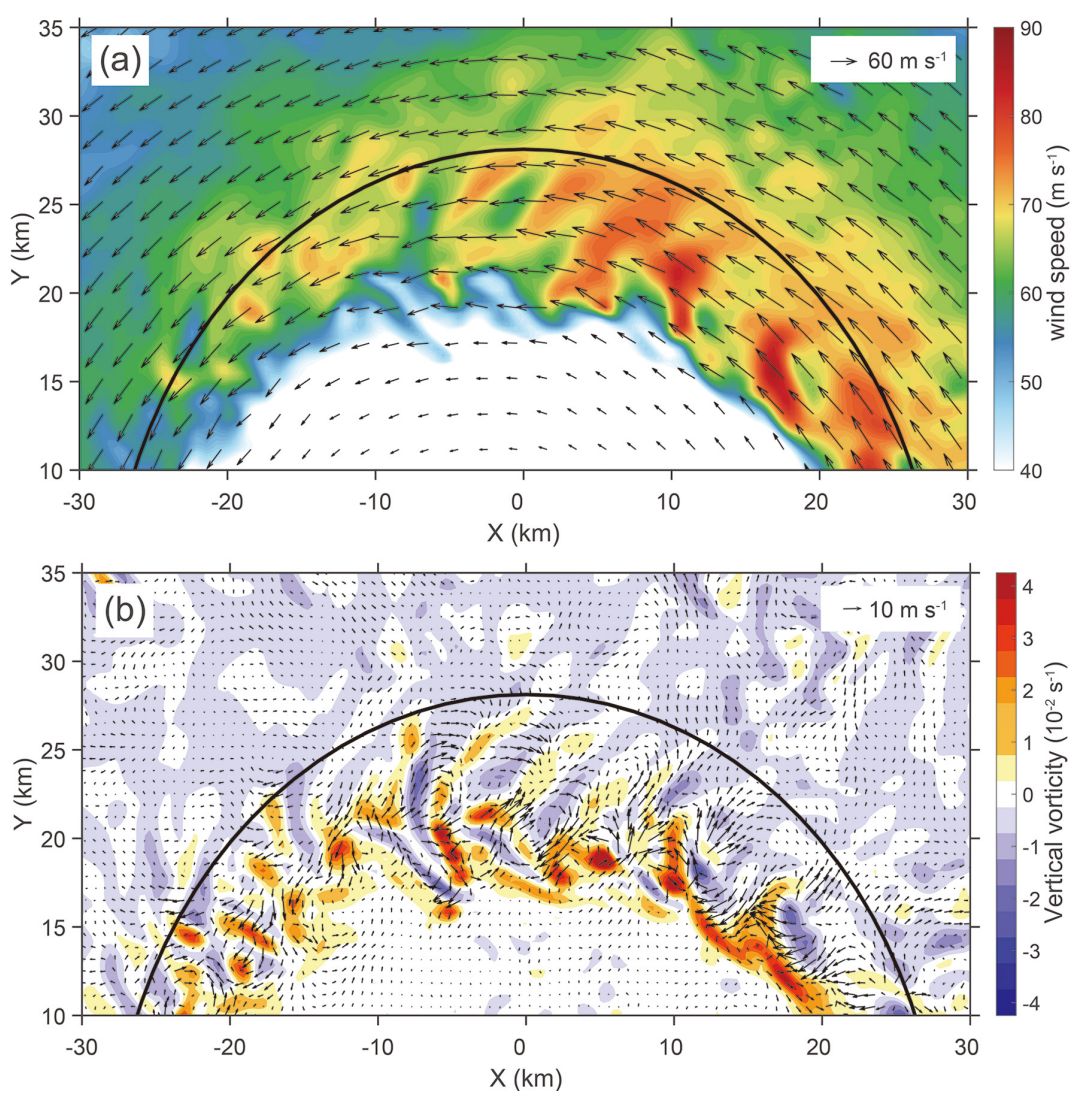

Fig. 7. (a) $0.5 \mathrm{~km}$ wind speed ( $\mathrm{m} \mathrm{s}^{-1}$, shading) and wind field ( $\mathrm{m} \mathrm{s}^{-1}$, vector) in the northern portion of the TC inner core at $24 \mathrm{~h} 45 \mathrm{~min}$ and (b) the corresponding perturbation wind field ( $\mathrm{m} \mathrm{s}^{-1}$, vector) obtained by subtracting wavenumber 0-3 components and vertical component of relative vorticity ( $\mathrm{s}^{-1}$, shading). The black line indicates the RMW.

features. To obtain the small-scale features, we subtract the wavenumber 0-3 components with respect to the TC center in the wind field (Fig. 7b). The smallscale disturbances are mainly located at the interface between the eye and eyewall, with diameters ranging from $1 \mathrm{~km}$ to several kilometers.

To demonstrate the impact of the small-scale features on the entrainment of the low-level high-entropy air from the eye to the eyewall, we calculated the forward trajectories of the air parcels initially in the TC eye by using the wavenumber $0-3$ components in the wind field. Figure 6 shows the comparison of the entrance azimuths of these air parcels at $0.5 \mathrm{~km}$ with those calculated with the original winds. Note that the entrance azimuth is defined as where an air parcel reaches the inner boundary of the eyewall before entering the eyewall region. We can see that the most active entrance location shifts cyclonically by about $30^{\circ}$ under the no-perturbation scenario, indicating the influence of the small-scale features on the entrainment. There are 624 air parcels enter the eyewall, accounting for $33.8 \%$ of the total (1846) air parcels. It is indicated that the small-scale disturbances increase the entrainment of the low-level high-entropy air from the eye to the eyewall by $5.3 \%$. Although our calculation is only based on a single case, it is suggested that the presence of the small-scale disturbances at the interface between the eye and the eyewall can enhance the entrainment of the low-level high-entropy air from the eye to the eyewall.

We should point out that the horizontal spacing of our numerical experiment marginally resolves the small-scale features at the interface between the eye and eyewall. We compare the small-scale features with those in the numerical experiment in Wu et al. (2018, 2019) and find that the small-scale features simulated in the present study are much weaker in terms of the associated updraft. In Wu et al. (2018, 2019), the hor- 


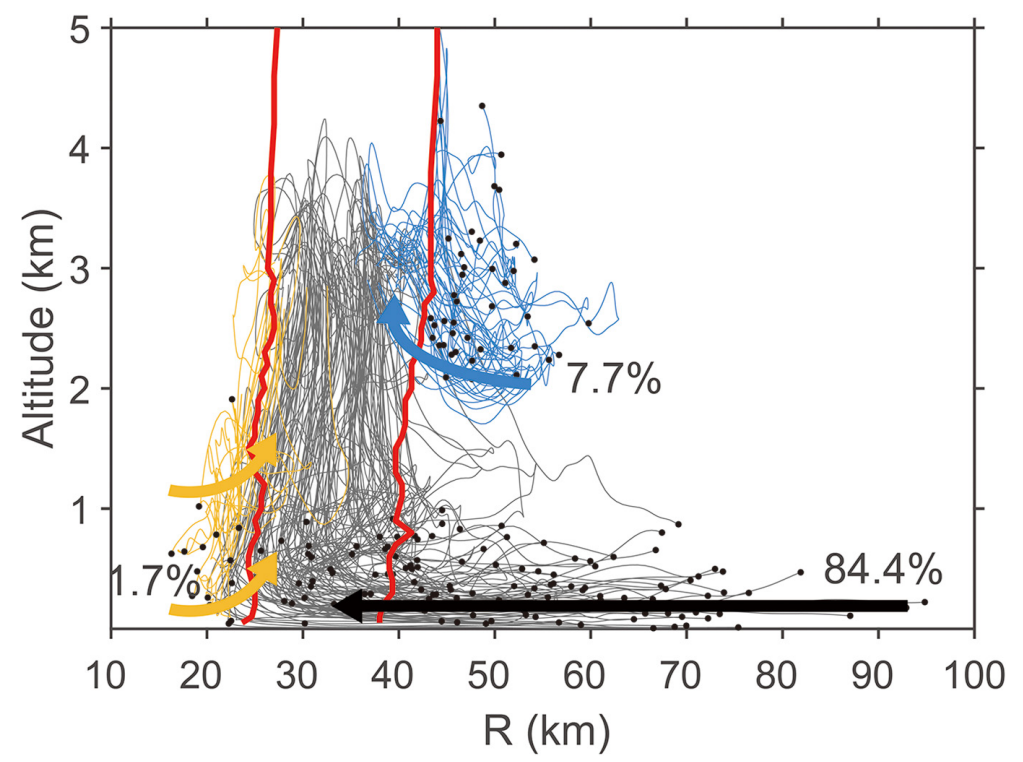

Fig. 8. A subset of the backward trajectories of the eyewall air parcels (184) that are initially at $3 \mathrm{~km}$. The colors of the trajectories indicate the three types of their origins: from the boundary inflow (black), the low-level eye (yellow), and middle-level environment (blue), which are also schematically shown by thick arrows. The solid lines denote the inner and outer boundaries of the eyewall with the dashed lines indicating the RMW.

izontal grid size is $37 \mathrm{~m}$. The influence of the smallscale features should increase when their strength and structure are more realistically simulated.

\section{Origins of the eyewall air parcels}

As we mentioned in the introduction, the influence of the entrainment of the low-level high-entropy air from the eye to the eyewall on TC intensity also depends on how many eyewall air parcels are from other origins. For this purpose, the original positions of air parcels in the eyewall are tracked by the calculation of the backward trajectories. As indicated in Table 1, the number of the eyewall air parcels ranges from 2291 to 3543 , much larger than the number of the eye air parcels below $3 \mathrm{~km}$.

In the last section, we already know that the entrainment of the low-level high-entropy air from the eye to the eyewall occurs only at the levels below 3 $\mathrm{km}$. For this reason, we take the eyewall region below $3 \mathrm{~km}$ as a whole and then evaluate the origins of the eyewall air parcels in the lower layer. Figure 8 shows a subset of the backward trajectories of the eyewall air parcels below $3 \mathrm{~km}$. The numbers of the trajectories are based on the percentage of each origin, whereas the individual trajectories are randomly selected in the subset. The figure just shows the three origins of the eyewall parcels outside the eyewall during the $90 \mathrm{~min}$ period. Besides the air parcels from the eye region, the eyewall parcels also come from the strong low-level inflow and the middle-level environment. The parcels from the middle-level environment are much drier than those in the eyewall with a mean $\theta_{e}$ of $352 \mathrm{~K}$ at the starting points. The entrainment of the middlelevel dry air has been known as the ventilation effect in previous studies, which tends to weaken TC intensity (Simpson and Rhiel 1958; Tang and Emanuel 2010).

The relative importance of the air parcels from the three origins can be quantified by calculating their percentages that account for all parcels entering the eyewall region below $3 \mathrm{~km}$ within the 90 min period. As expected, the eyewall parcels are dominantly from the low-level boundary inflow, accounting for $84.4 \%$ of the total incoming air parcels, whereas there are $7.7 \%$ of the air parcels from the middle-level dry environment. Although considerable air parcels in the low-level eye can be entrained into the eyewall, these parcels only account for $1.7 \%$ of the total incoming air parcels. It is suggested that the entrainment of the low-level high-entropy air from the eye to the eyewall has a negligible influence on the total eyewall air parcels below $3 \mathrm{~km}$ in terms of its percentage.

Following Cram et al. (2007), we can quantitatively examine the influence of the mixing of low-level high- 
entropy eye air to eyewall convection by calculating the change of $\theta_{e}$ along the trajectory as follows: $\Delta \theta$ $=\theta_{e}$ (eye or environment) $-\theta_{e}$ (eyewall). Since the backward trajectories are calculated, $\theta_{e}$ (eyewall) is the $\theta_{e}$ at the seed point and $\theta_{e}$ (eye or environment) is the $\theta_{e}$ at the first point of the trajectory in the eye or environment. For the parcels from the low-level high-entropy eye air (the boundary inflow), $(\overline{\Delta \theta})=$ $2.27 \mathrm{~K}(1.23 \mathrm{~K})$, indicating the mixing increases the $\theta_{e}$. For the entrainment of the dry environmental air parcels, $\overline{\Delta \theta}=-2.27 \mathrm{~K}$, indicating the mixing decreases the $\theta_{e}$. Note that the entrainment of the dry environmental air into the eyewall is not limited to the lower part of the eyewall. The dry air entrainment generally increases with altitude with two maxima at $6-8 \mathrm{~km}$ and around $12 \mathrm{~km}$ (figure not shown). The parcels from the low-level high-entropy eye air account for $5.8 \%$ of the total change in $\theta_{e}$ because of the small proportion in the incoming eyewall air parcels.

To further evaluate the relative importance of the entrainment of the low-level high-entropy eye air, we calculated the mass transport of various origins at 3 $\mathrm{km}$. In the calculation, each air parcel represents an area of $1 \times 1 \mathrm{~km}^{2}$. At $25.5 \mathrm{~h}$, the total mass transport of the eyewall is $28.8 \times 10^{5} \mathrm{~kg} \mathrm{~s}^{-1}$. The mass transport at $3 \mathrm{~km}$ height results dominantly from the boundary inflow and the ascending air that is originally in the eyewall. The former accounts for $79.2 \%$ of the mass transport $\left(22.8 \times 10^{5} \mathrm{~kg} \mathrm{~s}^{-1}\right)$, and the latter accounts for $14.2 \%$ of the mass transport $\left(4.1 \times 10^{5} \mathrm{~kg} \mathrm{~s}^{-1}\right)$. The two origins account for a total of $93.4 \%$, whereas the other two origins only account for $6.6 \%$ of the total mass transport. The air parcels from the lowlevel high-entropy reservoir account for $4.5 \%(1.3 \times$ $10^{5} \mathrm{~kg} \mathrm{~s}^{-1}$ ), and the air parcels from the dry environment account for $2.1 \%\left(0.6 \times 10^{5} \mathrm{~kg} \mathrm{~s}^{-1}\right)$.

We can see that the low-level high-entropy air from the eye also has little influence on the change of $\theta_{e}$ and mass transport in the eyewall. The results are generally consistent with the finding of Bryan and Rotunno (2009) and Wang and Heng (2016). Bryan and Rotunno (2009) found that a lack of the low-level high-entropy air in the TC eye can reduce the axisymmetric tangential wind speed by about $4 \%$ in their experiments. Wang and Heng (2016) found that the entrainment of the high-entropy eye air on TC intensity was not through the modifications to the overall strength of eyewall convection.

One may argue that the $90 \mathrm{~min}$ period is not long enough for quantifying the relative importance of the air parcels from different origins. The issue can be justified as follows. First, the same time period is used to calculate the entrainment of the eye air parcels into the eyewall. Second, the eyewall air parcels below $3 \mathrm{~km}$ are nearly recycled during the $90 \mathrm{~min}$ period, and only $6.2 \%$ parcels stay in the eyewall without an incoming origin. We should point out that the relative importance discussed here may change during different stages of TC lifetime.

\section{Summary}

Previous studies suggested that the entrainment of the high-entropy eye air is an additional energy source for TC intensification, likely responsible for the simulated superintensity of TCs (Persing and Montgomery 2003; Cram et al. 2007; Montgomery et al. 2006; Bell and Montgomery 2008), but Bryan and Rotunno (2009) demonstrated numerically that this effect is negligibly small. Although Cram et al. (2007) confirmed the entrainment of the high-entropy air below the inversion of the TC eye to the eyewall, their trajectory analysis did not quantitatively evaluate the role of the entrainment of the high-entropy eye air. In the present study, we used the 1 min output data from the high-resolution numerical simulation of a semi-idealized TC that evolves in a realistic largescale environment and evaluated the relative importance of the entrainment of the high-entropy eye air through the trajectory analysis.

In agreement with Cram et al. (2007), our analysis also indicates that considerable air parcels below the eye inversion can be entrained into the eyewall in the layer below $3 \mathrm{~km}$, and the entrainment occurs favorably on the quadrants of enhanced eyewall convection. Although the horizontal resolution of our simulation is relatively coarse for resolving the small-scale features at the interface between the eye and eyewall, we find that the presence of the small-scale disturbances can enhance the entrainment of the low-level high-entropy air from the eye to the eyewall. We speculate that the influence of the small-scale features on the entrainment may increase if their strength and structure are more realistically simulated.

Although there are considerable air parcels in the low-level eye that can be entrained into the eyewall in the 90 min period, these parcels can only account for $1.7 \%$ of the total eyewall air parcels. The eyewall air parcels below $3 \mathrm{~km}$ experience a fast recycling, with $84.4 \%$ of them from the low-level inflow and $7.7 \%$ of them from the middle-level dry environment, whereas only $6.2 \%$ air parcels stay in the eyewall without an incoming origin during the $90 \mathrm{~min}$ period. The parcels from the low-level high-entropy eye air account for $5.8 \%$ of the total change in $\theta_{e}$ because 
of the small proportion in the incoming eyewall air parcels. Calculation of the mass flux at $3 \mathrm{~km}$ height indicates that $79.2 \%$ of the mass transport results from the air parcels with the boundary layer inflow, whereas the air parcels from the low-level high-entropy reservoir account for $4.5 \%$ of the total mass transport. Consistent with the finding of Bryan and Rotunno (2009), the low-level high-entropy air from the eye has little direct influence on TC intensity by the calculation of the mass and thermodynamic contributions.

It should be pointed out that our evaluation is based on the direct contribution of the low-level high-entropy eye air to the eyewall convection. Studies indicated that the high-entropy air could have an indirect effect by enhancing localized strong updrafts in the eyewall, leading to the formation of convective bursts (CBs) (Hazelton et al. 2017) and promoting RMW contraction (Wang and Heng 2016). An analysis of the trajectories associated with CBs was conducted by using the method in Hazelton et al. (2017). It is found that $7.3 \%$ of the air parcels in CBs are from the low-level high-entropy eye air, suggesting that the entrainment may affect the TC intensity through prompting the CBs in the eyewall.

In the present study, we reconcile the results of Bryan and Rotunno (2009) and Cram et al. (2007). However, the robustness of the results of the present study deserves further investigation. First, the trajectory analysis is based on the model output over a 90 min period. Likely, the contribution of the entrainment of the low-level high-entropy air from the eye to the eyewall varies during the different stages of the TC development. Second, the horizontal resolution of our numerical experiment marginally resolves the smallscale features at the interface between the eye and the eyewall. The effect of the small-scale features may increase if their strength and structure are more realistically simulated.

\section{Acknowledgments}

The research was jointly supported by the National Natural Science Foundation of China (41730961, 41675051, 41675009, and 41905001) and the Open Research Program of the State Key Laboratory of Severe Weather (2019LASW-A02). The numerical simulation was conducted on Tianhe-2 Supercomputer in the National Supercomputer Center in Guangzhou, China. The authors also are grateful for the helpful editor of Prof. Wang Yuqing and three anonymous reviewers.

\section{Reference}

Aberson, S. D., M. T. Montgomery, M. Bell, and M. Black, 2006: Hurricane Isabel (2003): New insights into the physics of intense storms. Part II: Extreme localized wind. Bull. Amer. Meteor. Soc., 87, 1349-1354.

Aberson, S. D., J. A. Zhang, and K. N. Ocasio, 2017: An extreme event in the eyewall of Hurricane Felix on 2 September 2007. Mon. Wea. Rev., 145, 2083-2092.

Barnes, G. M., and P. Fuentes, 2010: Eye excess energy and the rapid intensification of Hurricane Lili (2002). Mon. Wea. Rev., 138, 1446-1458.

Bell, M. M., and M. T. Montgomery, 2008: Observed structure, evolution, and potential intensity of Category 5 Hurricane Isabel (2003) from 12 to 14 September. Mon. Wea. Rev., 136, 2023-2046.

Black, P. G., and F. D. Marks, 1991: The structure of an eyewall meso-vortex in Hurricane Hugo (1989). Proceedings of the 19th Conference on Hurricanes and Tropical Meteorology, Amer. Meteor. Soc., 579-582.

Braun, S. A., 2002: A cloud-resolving simulation of Hurricane Bob (1991): Storm structure and eyewall buoyancy. Mon. Wea. Rev., 130, 1573-1592.

Braun, S. A., M. T. Montgomery, and Z. Pu, 2006: Highresolution simulation of Hurricane Bonnie (1998). Part I: The organization of eyewall vertical motion. $J$. Atmos. Sci., 63, 19-42.

Bryan, G. H., and R. Rotunno, 2009: The influence of nearsurface, high-entropy air in hurricane eyes on maximum hurricane intensity. J. Atmos. Sci., 66, 148-158.

Cram, T. A., J. Persing, M. T. Montgomery, and S. A. Braun, 2007: A Lagrangian trajectory view on transport and mixing processes between the eye, eyewall, and environment using a high-resolution simulation of Hurricane Bonnie (1998). J. Atmos. Sci., 64, 1835-1856.

Duchon, C. E., 1979: Lanczos filtering in one and two dimensions. J. Appl. Meteor., 18, 1016-1022.

Emanuel, K. A., 1986: An air-sea interaction theory for tropical cyclones. Part I: Steady-state maintenance. $J$. Atmos. Sci., 43, 585-605.

Emanuel, K. A., 1995: Sensitivity of tropical cyclones to surface exchange coefficients and a revised steadystate model incorporating eye dynamics. J. Atmos. Sci., 52, 3969-3976.

Emanuel, K. A., 1997: Some aspects of hurricane inner-core dynamics and energetics. J. Atmos. Sci., 54, 10141026.

Frank, W. M., and E. A. Ritchie, 1999: Effects of environmental flow upon tropical cyclone structure. Mon. Wea. Rev., 127, 2044-2061.

Hazelton, A. T., R. F. Rogers, and R. E. Hart, 2017: Analyzing simulated convective bursts in two Atlantic hurricanes. Part I: Burst formation and development. Mon. Wea. Rev., 145, 3073-3094.

Hong, S.-Y., and J. O. J. Lim, 2006: The WRF single-moment 6-class microphysics scheme (WSM6). J. Korean 
Meteor. Soc., 42, 129-151.

Ito, J., T. Oizumi, and H. Niino, 2017: Near-surface coherent structures explored by large eddy simulation of entire tropical cyclones. Sci. Rep., 7, 3798, doi:10.1038/ s41598-017-03848-w.

Jordan, E. S., 1952: An observational study of the upper wind-circulation tropical storms. J. Meteor., 9, 340346.

Kain, J. S., and J. M. Fritch, 1993: Convective parameterization for mesoscale models: The Kain-Fritch scheme. The Representation of Cumulus Convection in Numerical Models. Meteor. Monogr., No. 24, Amer. Meteor. Soc., 165-170.

Knaff, J. A., J. P. Kossin, and M. DeMaria, 2003: Annular hurricanes. Wea. Forecasting, 18, 204-223.

Kossin, J. P., B. D. McNoldy, and W. H. Schubert, 2002: Vortical swirls in hurricane eye clouds. Mon. Wea. Rev., 130, 3144-3149.

Marks, F. D., P. G. Black, M. T. Montgomery, and R. W. Burpee, 2008: Structure of the eye and eyewall of Hurricane Hugo (1989). Mon. Wea. Rev., 136, $1237-$ 1259.

Mirocha, J. D., J. K. Lundquist, and B. Kosović, 2010: Implementation of a nonlinear subfilter turbulence stress model for large-eddy simulation in the Advanced Research WRF model. Mon. Wea. Rev., 138, 4212-4228.

Miyamoto, Y., and T. Takemi, 2013: A transition mechanism for the spontaneous axisymmetric intensification of tropical cyclones. J. Atmos. Sci., 70, 112-129.

Montgomery, M. T., M. M. Bell, S. D. Aberson, and M. L. Black, 2006: Hurricane Isabel (2003): New insights into the physics of intense storms. Part I: Mean vortex structure and maximum intensity estimates. Bull. Amer. Meteor. Soc., 87, 1335-1347.

Noh, Y., W. G. Cheon, S.-Y. Hong, and S. Raasch, 2003: Improvement of the K-profile model for the planetary boundary layer based on large eddy simulation data. Bound.-Layer Meteor., 107, 401-427.

Persing, J., and M. T. Montgomery, 2003: Hurricane superintensity. J. Atmos. Sci., 60, 2349-2371.

Simpson, R. H., and H. Riehl, 1958: Mid-tropospheric ventilation as a constraint on hurricane development and maintenance. Proceedings of the Technical Conference on Hurricanes, Amer. Meteor. Soc., D4-1-
D4-10.

Stern, D. P., and D. S. Nolan, 2012: On the height of the warm core in tropical cyclones. J. Atmos. Sci., 69, 1657-1680.

Stern, D. P., and F. Zhang, 2013: How does the eye warm? Part II: Sensitivity to vertical wind shear and a trajectory analysis. J. Atmos. Sci., 70, 1849-1873.

Tang, B., and K. Emanuel, 2010: Midlevel ventilation's constraint on tropical cyclone intensity. J. Atmos. Sci., 67, 1817-1830.

Wang, Y., and J. Xu, 2010: Energy production, frictional dissipation, and maximum intensity of a numerically simulated tropical cyclone. J. Atmos. Sci., 67, 97-116.

Wang, Y., and J. Heng, 2016: Contribution of eye excess energy to the intensification rate of tropical cyclones: A numerical study. J. Adv. Model. Earth Syst., 8, 1953-1968.

Willoughby, H. E., 1998: Tropical cyclone eye thermodynamics. Mon. Wea. Rev., 126, 3053-3067.

Wu, L., and X. Chen, 2016: Revisiting the steering principal of tropical cyclone motion in a numerical experiment. Atmos. Chem. Phys., 16, 14925-14936.

Wu, L., Q. Liu, and Y. Li, 2018: Prevalence of tornado-scale vortices in the tropical cyclone eyewall. Proc. Natl. Acad. Sci. U. S. A., 115, 8307-8310.

Wu, L., Q. Liu, and Y. Li, 2019: Tornado-scale vortices in the tropical cyclone boundary layer: Numerical simulation with the WRF-LES framework. Atmos. Chem. Phys., 19, 2477-2487.

Wurman, J., and K. Kosiba, 2018: The role of small-scale vortices in enhancing surface winds and damage in Hurricane Harvey (2017). Mon. Wea. Rev., 146, 713722.

Xu, J., and Y. Wang, 2010a: Sensitivity of tropical cyclone inner-core size and intensity to the radial distribution of surface entropy flux. J. Atmos. Sci., 67, 1831-1852.

$\mathrm{Xu}$, J., and Y. Wang, 2010b: Sensitivity of the simulated tropical cyclone inner-core size to the initial vortex size. Mon. Wea. Rev., 138, 4135-4157.

Yang, B., Y. Wang, and B. Wang, 2007: The effect of internally generated inner-core asymmetries on tropical cyclone potential intensity. J. Atmos. Sci., 64, 11651188. 